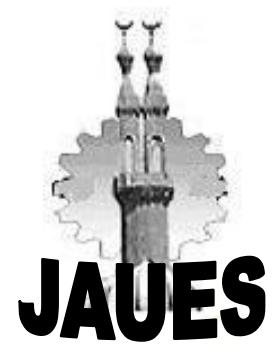

Journal Of Al Azhar University Engineering Sector

Vol. 12, No. 42, January, 2017, 395-412

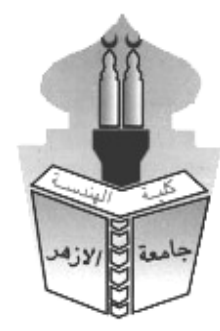

\author{
نحو مدن ذكية ذات كفاءة وظيفية \\ دراسة تطبيقية علي مشروع مدينة المستقبل بالعاصمة التشيكية براغ فئة

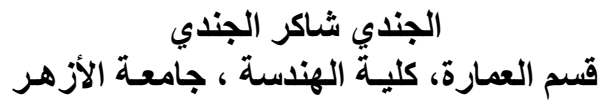

\begin{abstract}
Recent years have witnessed problems in the field of energy, and quality of life. in scope of technical progress and continuous increase in population here is come the need to create smart cities, by develop the existing cities to become smart cities, or build new cities that established from the beginning using sophisticated technological systems. The study cover smart cities from many aspects, the researcher address some important topics in smart cities, also point to some applications that could be implemented in the existing cities to develop services for people and enhance the quality of life and to provide a sustainable digital environment that become friendly to environment and encourage for learning and creativity to achieve happiness and welfare.

The study explains some basic standard criteria for smart city, we apply these standard criteria on the Future City (which the researcher was one of its design team and make main role in the final design) to see how much this city achieve these standard criteria and list these results in the end of the research. The researcher follows in this research the inductive, analytical and practical approach to learn about the role of smart cities in achieving progress and welfare of the citizens.
\end{abstract}

Keywords : Smart Cities, Sustainability, functional efficiently, Quality of life.

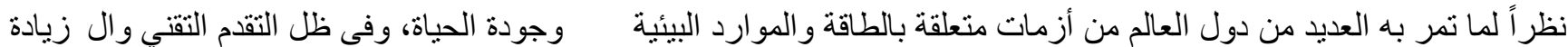

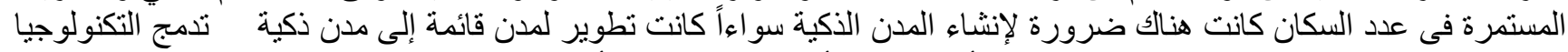

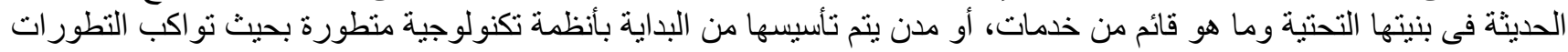

وتأتي أهمية البحث في إطار تناوله لأحد الموضو عات الهامة؛ حيث يتناول دراسة المدن الذكية من العديد من الجوانب، كما

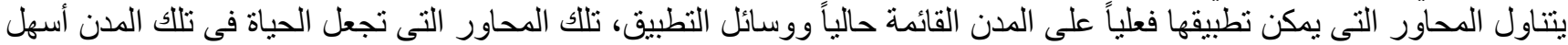

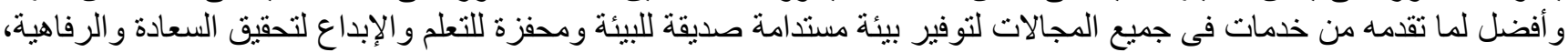

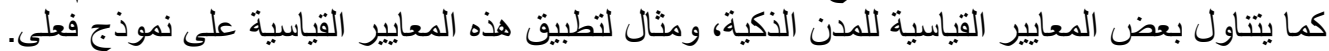

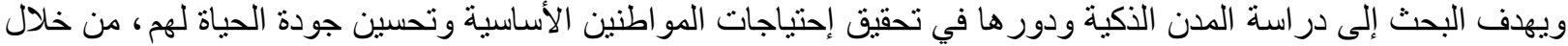

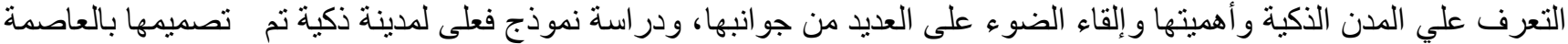

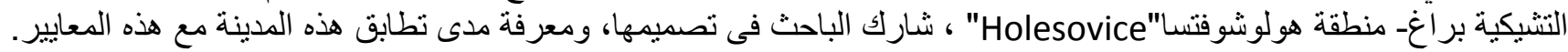

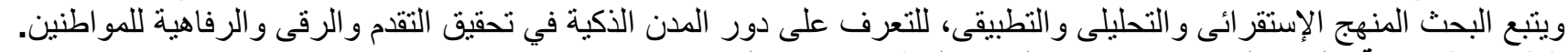

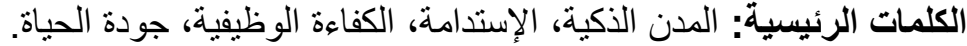

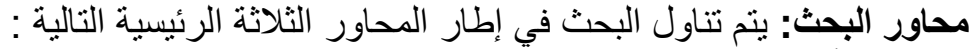

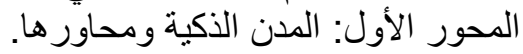

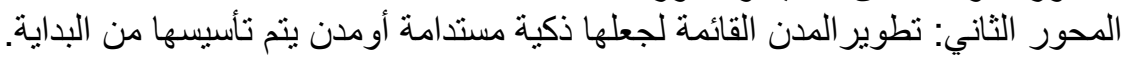
المحور الثالث: تطبيق بعض معايير المدن الذكية على مثال فعلى والتأكد من نجاحهـ. 


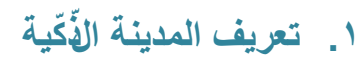

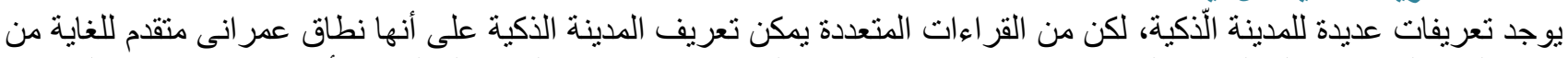
حيث البنية التحتية شاملة المبانى المستدامة، الإتصالات و طرق التسوق، وتعتبر تكنولوجيا المعلومات أساس بنائها وتقديم الخدمات

الرئيسية لسكانها.

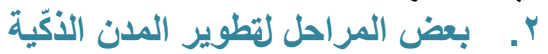

هناك بعض المر احل و الحلول للوصول إلي مدينة ذكّية (شكل رقم 1) منها مايلي (Ruthbea 2013):

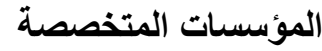

ويقصد بها بعض المؤستسات القائمة على التخطيط الجيد.

إستغلال الفرص المتاحة (Opportunistic)

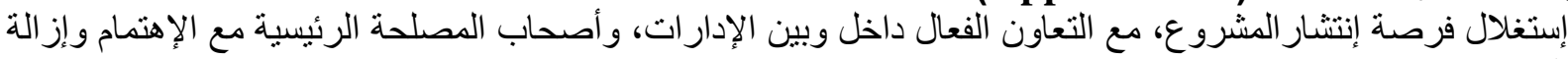
العوائق.

(القابلية للتكرار(ايقي (Repeatable) إستكمال المشاريع مع فاعليتها لتحقيق التكامل مع إمكانية تكر ار النموذج المتكامل.

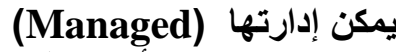

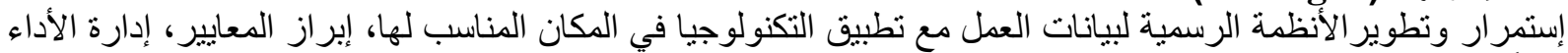

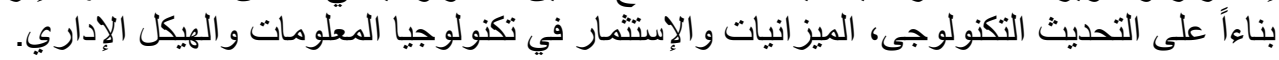

الوصول إلى الحد الأمثل (Optimized)

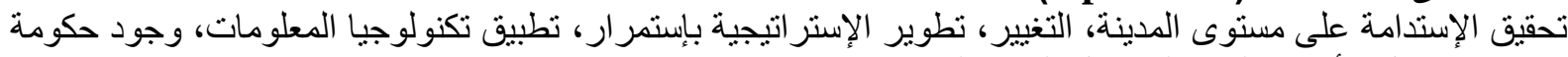
تسمح بإستقلال الأنظمة المتكاملة داخل النظام العام.

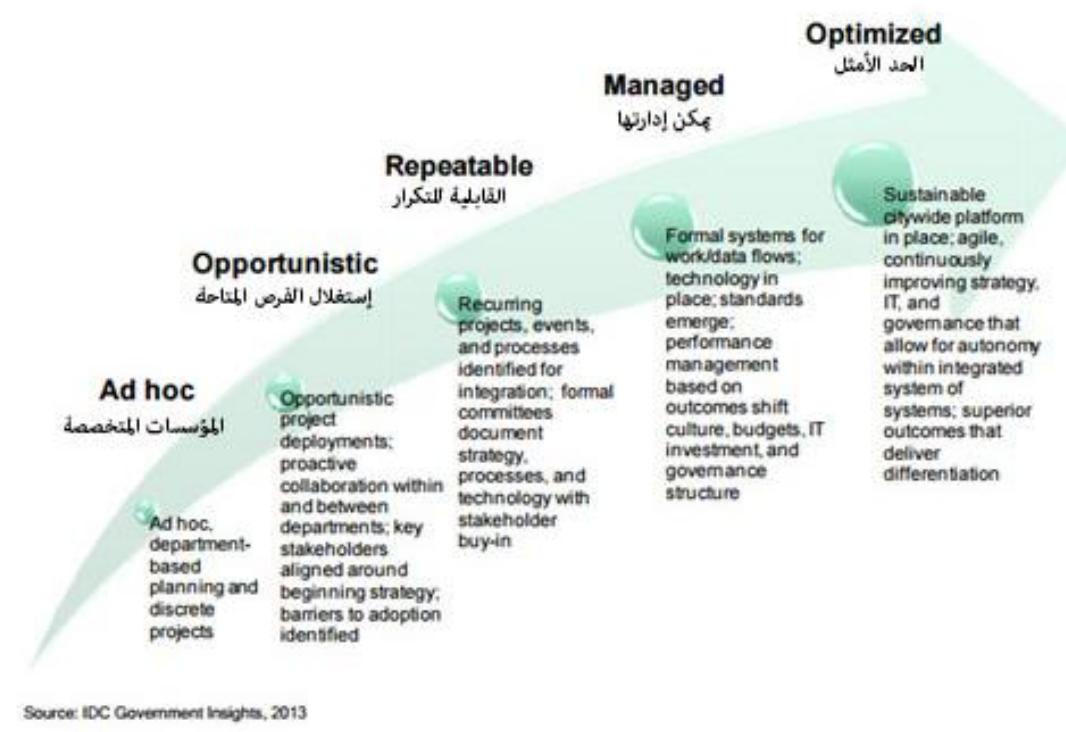

شكل (1) بعض المراحل والحلول للوصول إلي مدينة ذكية

Smart Cities and the Internet of Everything , 2013 المصدر :

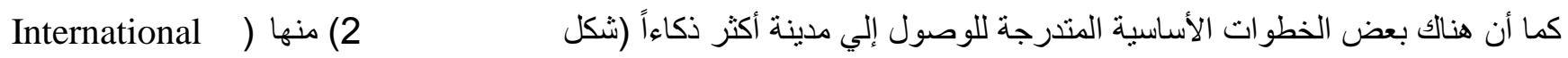
:(Electrotechnical Commission IEC 2014 
شكل (2 ) خطوات متدرجة لمدينة أكثر ذنَاءاً

Orchestrating infrastructure for sustainable Smart Cities , 2014 :

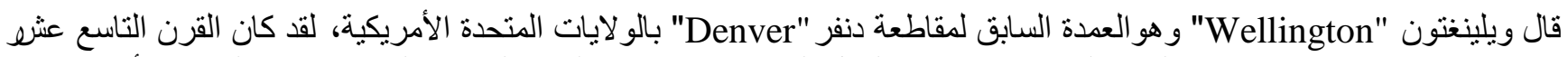

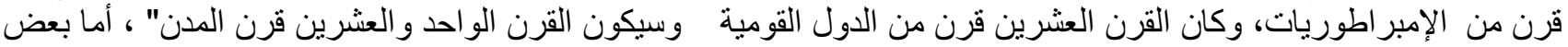

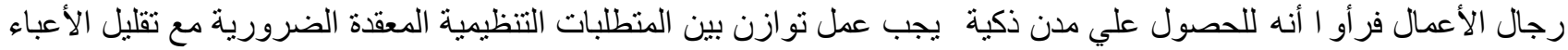


ل مد خطوط الملكية: منح المستأجر الإنتفاع بالفر اغ الموجود أمامه مدا يعطى الإحساس بالمسؤلية بالإهتمام باء.

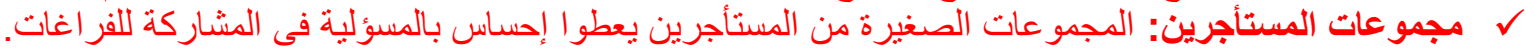

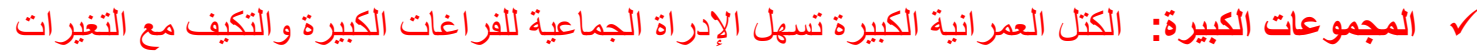

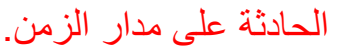
ل تعاون الجيران: يساعد السكان على إدارة الأماكن العامة (المدارس، المنتزهات، الأسواق التجارية) و يساعدوا في تغيير هذه الأماكن عند الحاجة.

ل المبانى الصناعية (المصانع) الغير مستخدمة من الممكن إعادة إستخدامها.

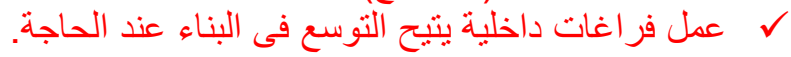
• - م القضايا المطروحة ل التركيبة السكانية و علاقاتها بالدخل و العمر. ل - ل

تقليل إنبعاثات الكربون عن طريق إستخدام الطاقة المتجددة و عدد من إستراتيجيات توليد الطاقة مثل الوقود الحيوى، الطاقة

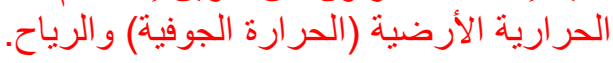

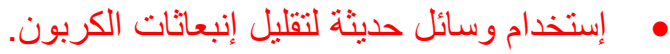

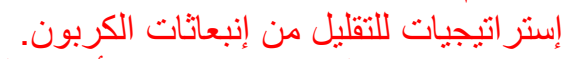
ل إدارة النفايات (إستخدام الأسمدة البيولوجية ـ حرق النفايات للوصول إلى مصدر إضافى للطاقة ــ التدوير قدر الإمكان).

ل إدارة المياة (إعادة تدوير المباة المستخدمة ـ تقليل المباة المهدرة عن طريق التوان عية و تغيير أنماط الحياة).

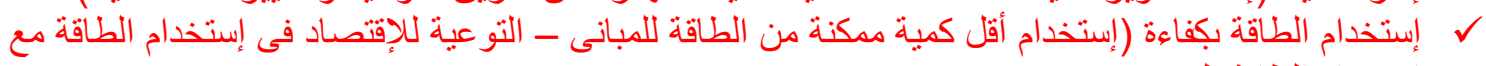
إستخدام الطاقة المتجددة ).

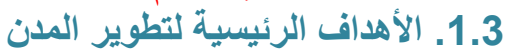

هناك العديد من الأهداف العامة لتطوير المدن لتصبح ذكية منها (Alan, Nguyen et al. 2006):

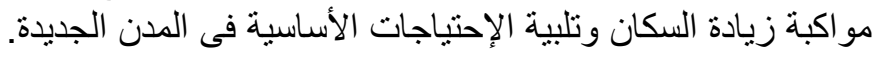
تحقيق التوازن بين الإستثمار و التنمية.

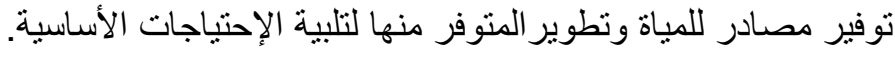

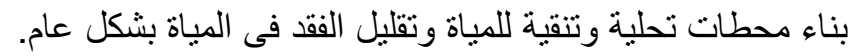

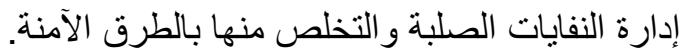

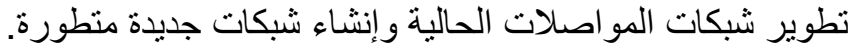

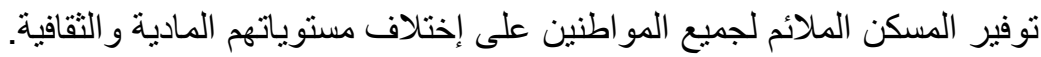
تطوير البنية التحتية و إنشاء شبكات جديدة تو اكب التوسعات التوات المحتملة. إنشاء مصارف خاصة بالفياضانات و الأمطار لتجنب الكوارة توارث التبات البيئية.

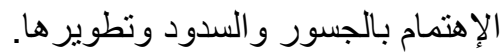

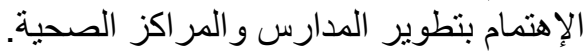
تطوير مر اكز المدن و الإهتمام بالأماكن العامة والمانة الترفيهية.

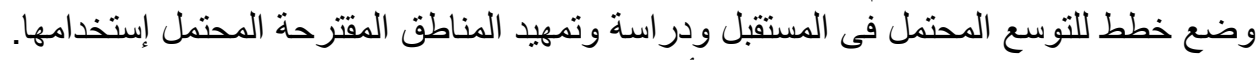

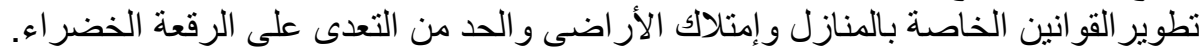

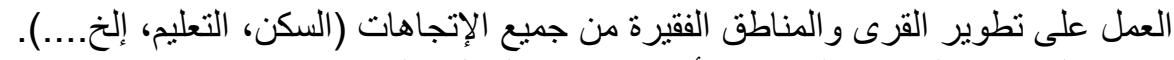

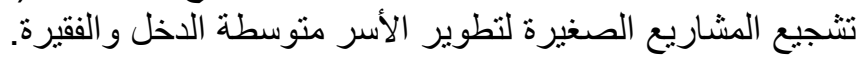

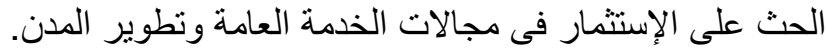

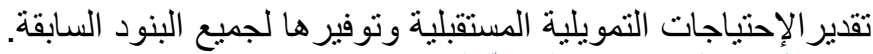

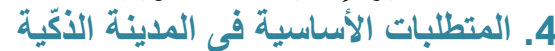
هناك بعض المتطلبات الأساسية فى المدينة الذكيّة أهمها (Macmillan 2004) : إمدادات كافية من المباة. 


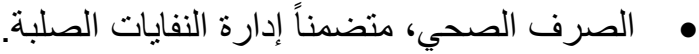

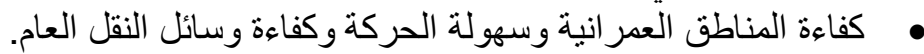

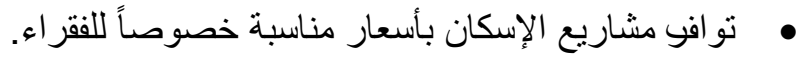

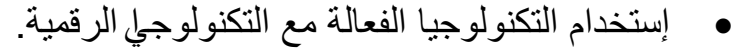

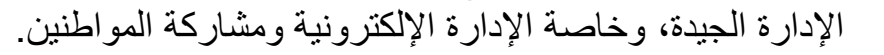

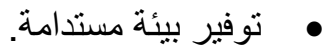

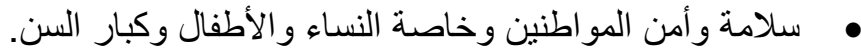

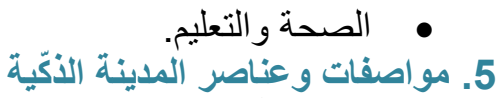

للمدن الذكية سواءاً كانت قائمة اومقترحة بعضة الأكضة المواصفة اصفات مثل (Giffinger, Fertner et al. 2007):

1.5 الإقتصاد الأكّى (التنافسية)

$$
\begin{aligned}
& \checkmark \\
& \checkmark \\
& \checkmark \\
& \checkmark \\
& \checkmark \\
& \checkmark
\end{aligned}
$$

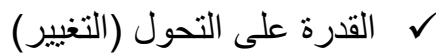

2.5. مواطنين مؤهلين للمدن الذكية (رأس المال للفرد والفير) المجتمع)

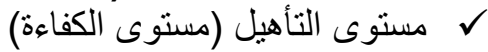
ل

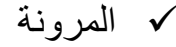
ل الإبداع $\checkmark$ ل

3.5. الحكومة الأكية (المشاركة)

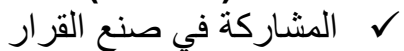

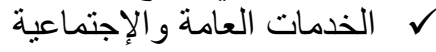

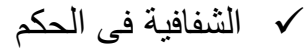

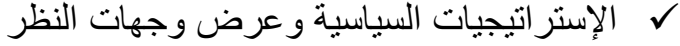

4.5. النقل الأكى (النقل بإستخدام تكنولوجيا المعلومات والإتصالات) ل $\checkmark$ ل

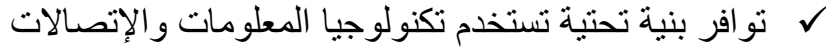
ل

5.5. البيئة الأكية (مصادر طبيعية)

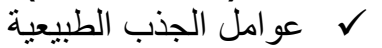
ل الظروف و الأحوال الجوية ح ل حماية البيئة 、 $\checkmark$

6.5. الحياة الأكّية أو العيش بذكاء (جودة الحياة)

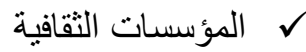
$\checkmark$ 


$$
\begin{aligned}
& \text { السلامة الفردية (سلامة الأفراد) } \\
& \text { جو } \\
& \checkmark \\
& \checkmark \\
& \checkmark
\end{aligned}
$$

\begin{tabular}{l} 
SMART ECONOMY \\
(Competitiveness) \\
\hline : Innovative spirit \\
: Entrepreneurship \\
: Pronomic image \& trademarks \\
: Intexibility of labour market \\
\end{tabular}

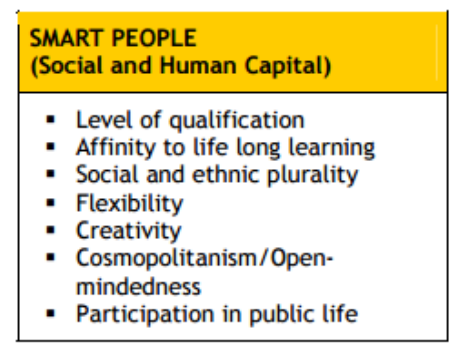

\section{SMART GOVERNANCE}

(Participation)

- Participation in decision-making

- Public and social services

- Transparent governance

- Political strategies \&

perspectives

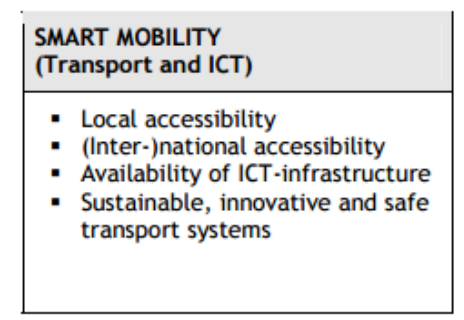

\section{SMART ENVIRONMENT (Natural resources)}

- Attractivity of natural conditions

- Pollution

- Environmental protection

- Sustainable resource management

\begin{tabular}{|l|}
\hline SMART LIVING \\
(Quality of life) \\
\hline - Cultural facilities \\
- Health conditions \\
- Housinidual safety \\
- Education facilities \\
- Touristic attractivity \\
\hline
\end{tabular}

$$
\text { شكل (4) أهم مواصفات وعناصر المدينة الأكية }
$$

المصدر:Smart cities, Ranking of European medium-sized cities, (2007)

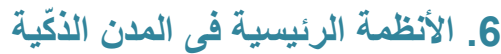

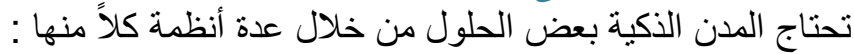

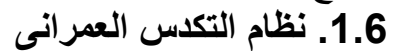

$$
\begin{aligned}
& \checkmark \text { لكدس الناس حول المناطق الهامة مع قلة المناطق الخضر اءء. }
\end{aligned}
$$

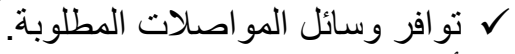

$$
\begin{aligned}
& \checkmark \\
& \checkmark \text { ل إرتفاع فى أسعار الوحدات السكنية. }
\end{aligned}
$$

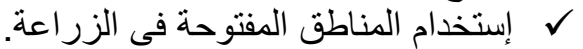

$$
\begin{aligned}
& \text { 2.6. نظام العودة إلى الطبيعة } \\
& \checkmark \\
& \text { ل زيادة المساحة المحددة للعائلة و إتاحة الفرصة لانئل للأفر اد للعيش بمفردهم. }
\end{aligned}
$$

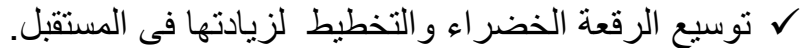

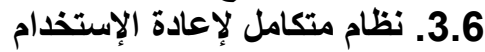

$$
\begin{aligned}
& \text { ل إصلاح المبانى القديمة و إعادة إستخدامها. }
\end{aligned}
$$

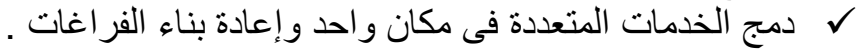

$$
\begin{aligned}
& \text { ل إنتاج الطاقة وإعادة إستخدام المبانى يشجع على وجود نشاطات جديدة. }
\end{aligned}
$$




$$
\begin{aligned}
& \text { 4.6. نظام مرن عملى ومتكيف التفو } \\
& \text { ل إستخدام مصانع التدوير للإستفادة من المخلفات داخل المدن. }
\end{aligned}
$$

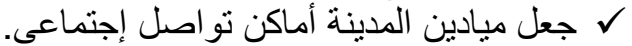

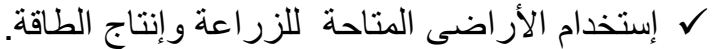

$$
\begin{aligned}
& \text { 5.6. نظام متظور وصديق للبيئة } \\
& \text { ل } \\
& \text { ل الحفاظ على الأراضى من أجل الزر اعة و إنتاج الطاقة. } \\
& \checkmark
\end{aligned}
$$

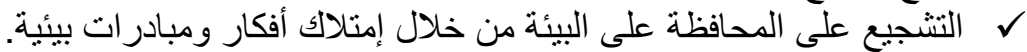

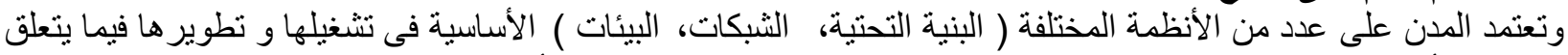

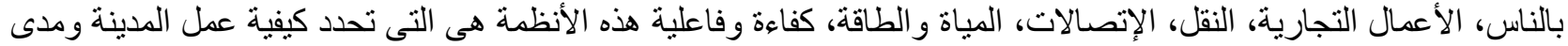

نجاحها فى تحقيق أهدافها (Services, 2009).

السكان: يشير نظام السكان داخل المدينة إلى الثبكات السكانية والثانية الإجتماعية. وتشمل السلامة العامة (الإطفاء، الثرطة

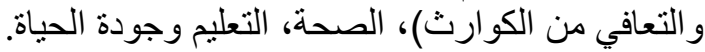

الأعمال: نظام العمل بالمدينة ويشمل التنظيم و السياسة البيئية، ويتضمن أنظمة التخطيط و الإنفتاح على التجارة الخارجية

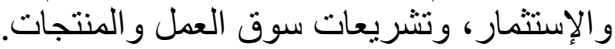

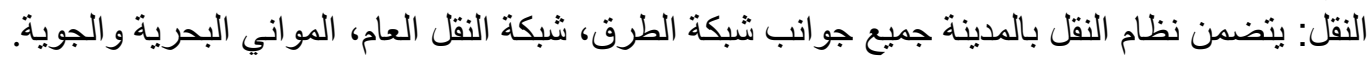

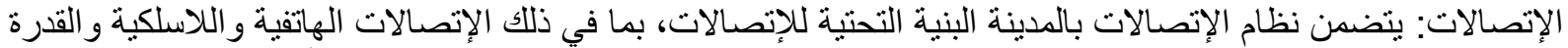

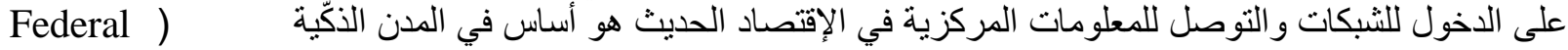

(Ministry of Economics and Technology 2010

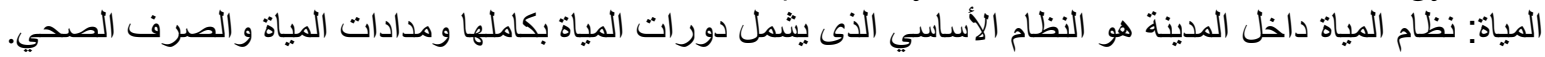

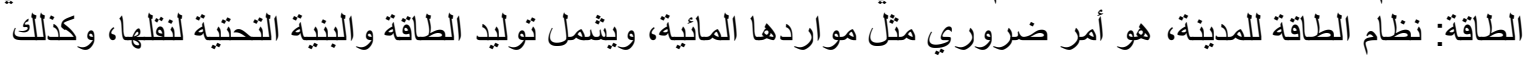

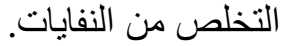
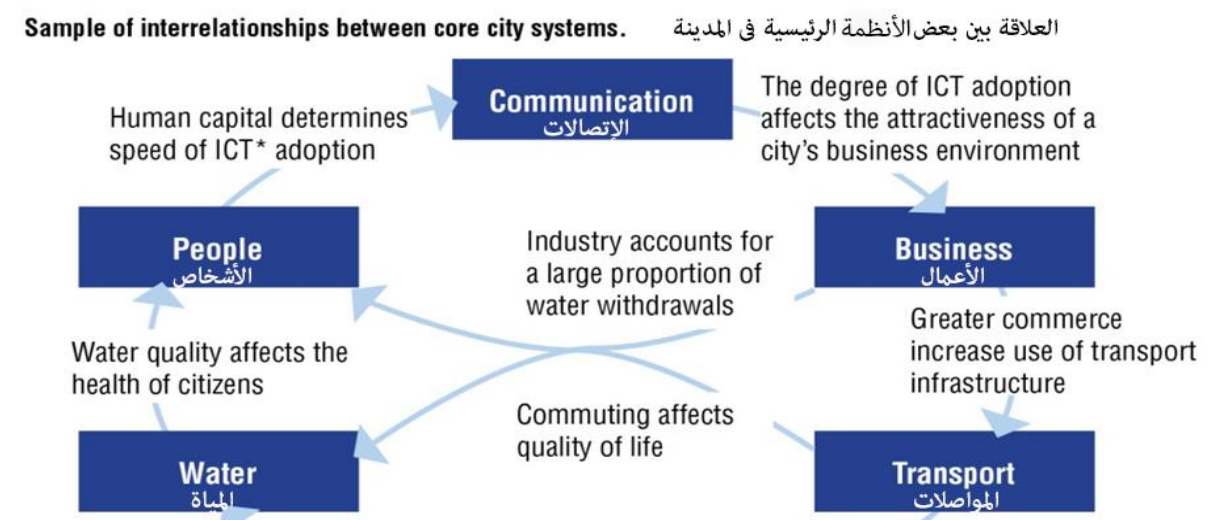

Energy is the reason for a substantial part of all water withdrawals

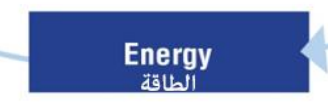

Transportation is one of the primary consumers of energy demand

$$
\text { شكل (3) يوضح العلاقة بين الأنظمة الرئيسية فى المدن الأكّية }
$$

المصدر:A vision of smarter cities, (2009)

هذه النظم الأساسية منر ابطة ويجب أن تعامل كنظام واحد يربط بين هذه الأنظة لجعلها تعمل بشكل أفضل. 


\section{نحو مدن ذكية ذات كفاءة وظيفية دراسة تطبيقية علي مشروع مدينة المستقبل بالعاصمة التشيكية بر اغ}

يجب إستخدام كل من الموارد البشرية والطبيعية و الطاقة والمياة بصورة متكاملة للوصول إلى حلول مرضية .(www.viralvibes.net)

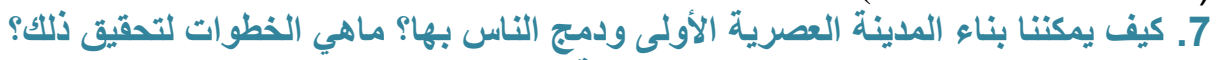

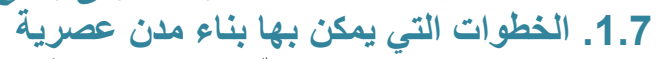

توفير المعلومات اللاّزمة التي من شأنها أن تساعد في تطوير ثقافة عالمية مستدامة من خلال توفير وسائل لتسخير مصادر

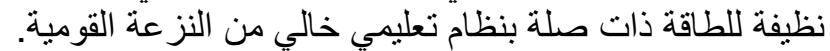

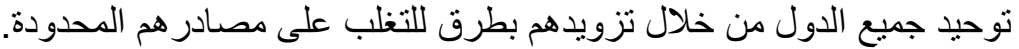

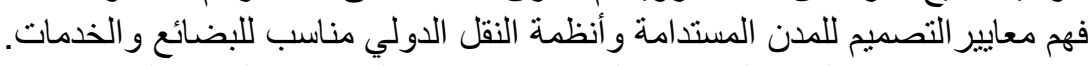

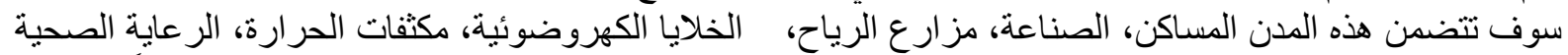

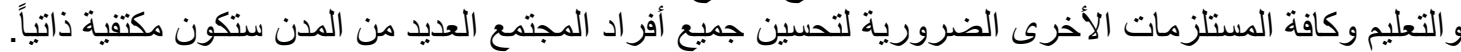

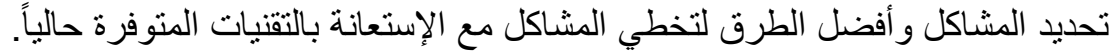

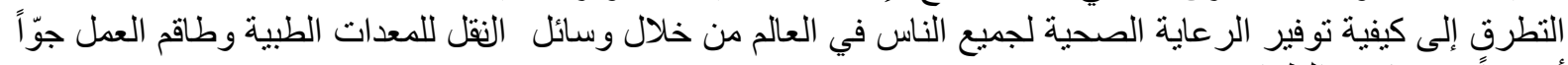
أوبحر أ في حالات الطو الطوارئ.

توفير أيضنا الطرق وعمليات تحلية المياة بدون إسنتخدام الوقود الأحفوري عن طريق مُكئفات حر ارية، نظم ألو اح ضوئية

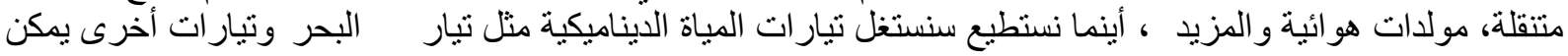

توجيهوا نحو مجال تطوير الطاقة.

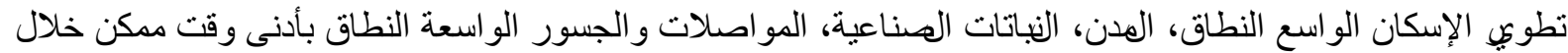
إستعمال العناصر البنيوية المقوّاة.

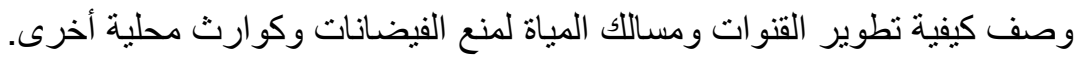

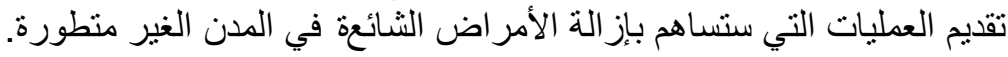
تقديم حلول ومنهجية لتنفيذ ثقافة عالمية موحدة مستدامة.

بناء مدن جديدة فقط لن يحقق التغيير ات المطلوبة دون إعداد أثخاص بتوجة بئه ملائم للتحرك للأمام وبدون القيم اللاّزمة لتحقيق ذلك.

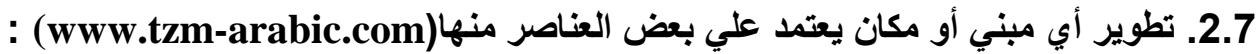
1 ـ الموقع ومدي أهميته ودور المعماري في هذي هذا هو الإستغلال الأمثل لهذا الموقع ومكانته و إمكانياته في تحديد النشاط الأمثل له

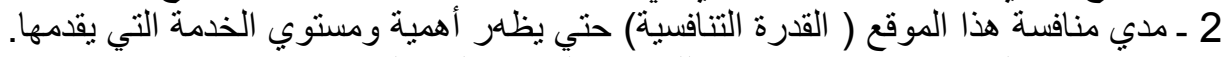

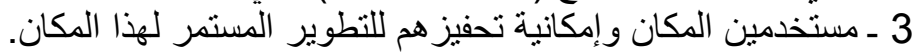

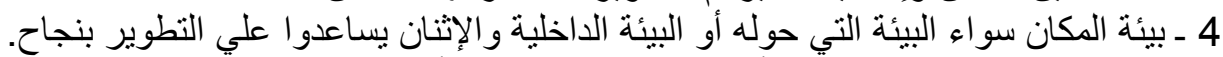

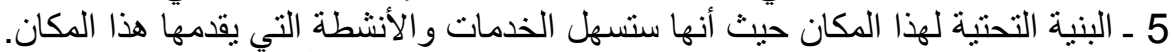

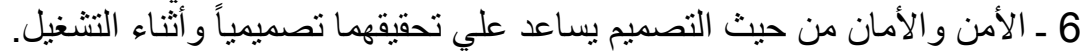

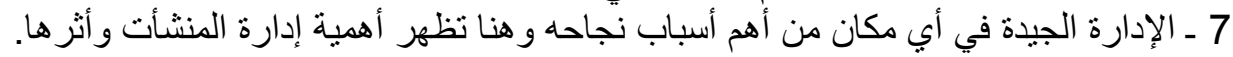

8 - التنمية المكانية.

8. خطوات تصميم المدن الأكيّة

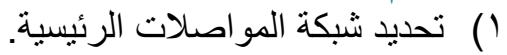

r) (Y) تحديد المناطق التجريبية (demo zones).

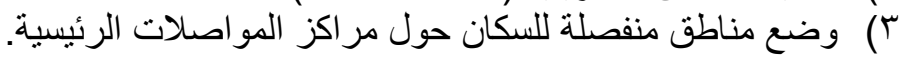

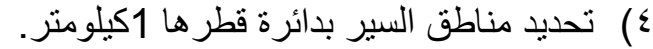

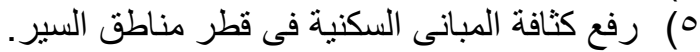

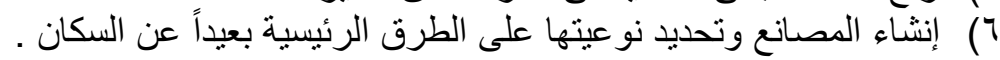

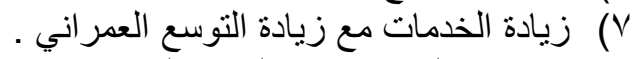

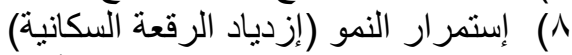

9 ) إنعكاس التوسع العمر اني علي قلة أوزيادة التعداد النعاد السكاني

9. بعض خواص المجتمعات المستدامة (بيئياً، إجتماعياً وإقتصادياً) ل الإستخدام الأمثل للفر اغات. 


$$
\begin{aligned}
& \text { ل }
\end{aligned}
$$

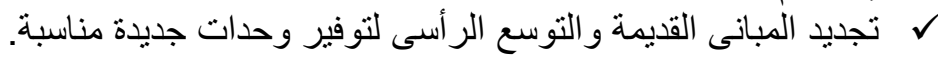

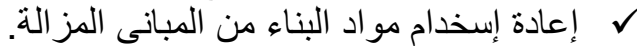

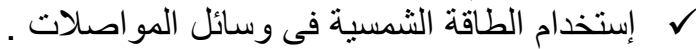

$$
\begin{aligned}
& \text { ل }
\end{aligned}
$$

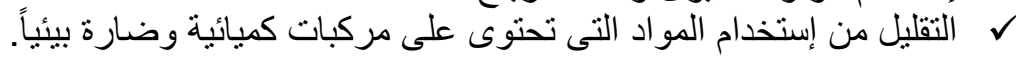
ل

ل لشر الوعى و التعريف بالمجتمعات المستدامة والعادات البات البيئية الصحيحة.

ل لشر الوعى بأهمية المواد المحلية لتقليل الإستير اد.

ل

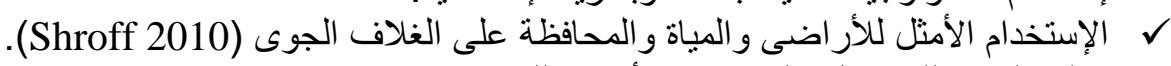

ح تحلية المياة (للحصول على مصادر أخرى للمياة).

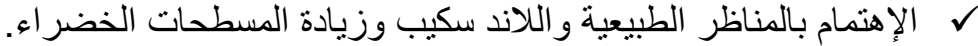

ل

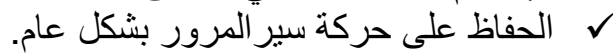

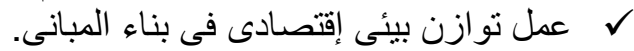

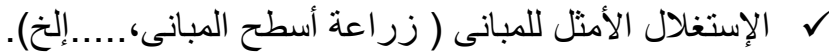

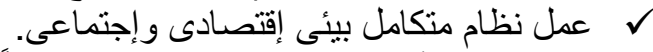

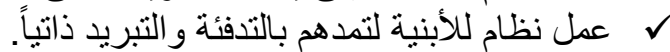

ل

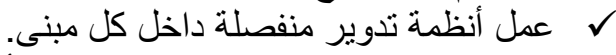

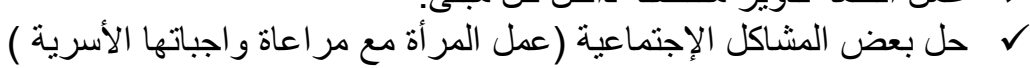

ل توفير قاعدة بيانات لمتابعة التطور وحل الماعلة المشكلات.

ل

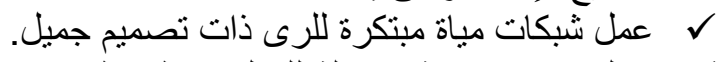

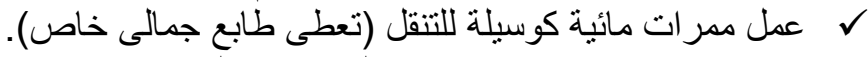

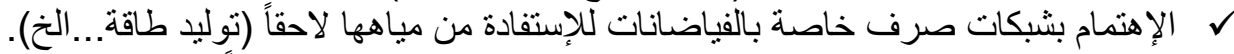

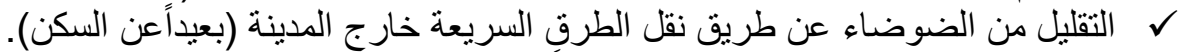

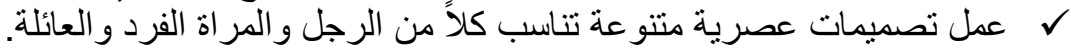

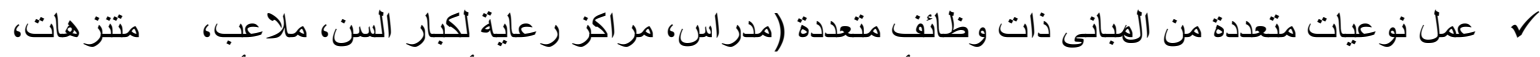

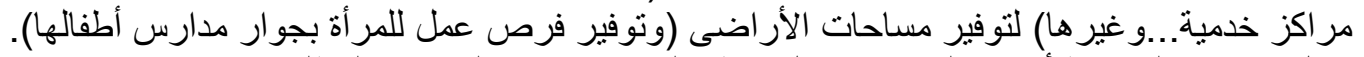

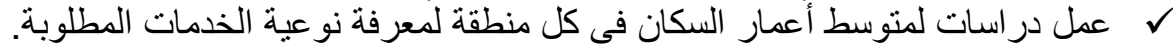

ل

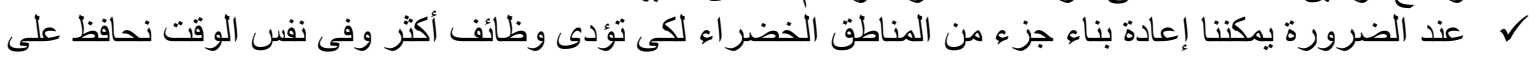

جزء منها .

ل

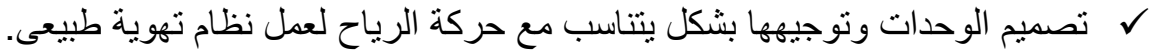

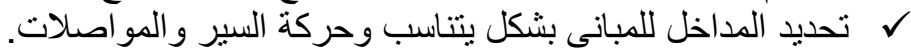

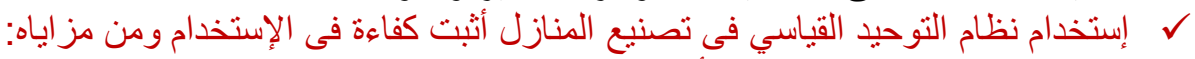

* تقليل تكلفة البناء (البناء بتكلفة أقل).

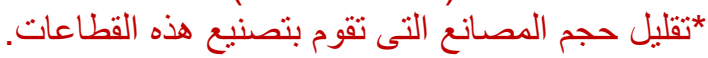

*تقليل الوقت المستهلك فى البناء.

*توفير للطاقة مع إستخدام التقنيات الحديثة.

10. بعض المشاكل فى المدن القديمة

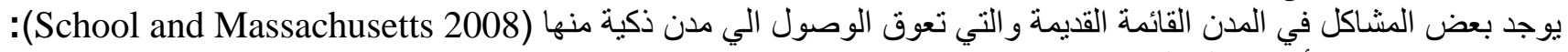
ل الأزمات المحلية. 
نحو مدن ذكية ذات كفاءة وظيفية در اسة تطبيقية علي مشروع مدينة المستقبل بالعاصمة التثيكية براغ

$$
\begin{aligned}
& \text { ل ع ع رداءة المبانى القائمة. }
\end{aligned}
$$

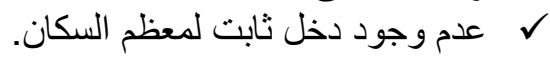

$$
\begin{aligned}
& \text { ل المنافسة الصناعية و الإقتصادية العالمية. }
\end{aligned}
$$

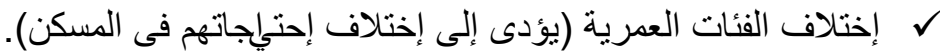

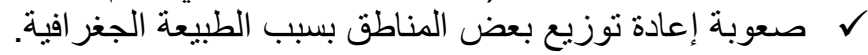

$$
\begin{aligned}
& \checkmark
\end{aligned}
$$

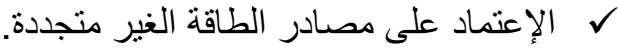

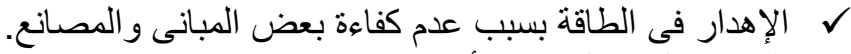

$$
\begin{aligned}
& \text { ل }
\end{aligned}
$$

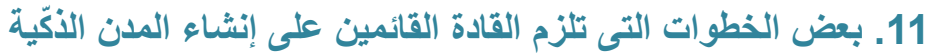

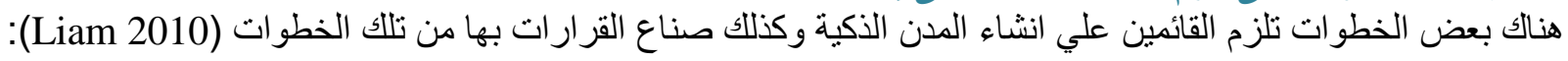

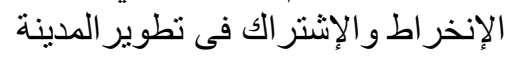
توضيح الرؤى المطلوبة الإنية

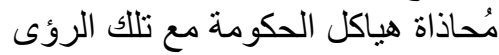

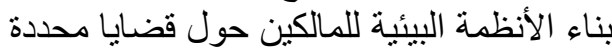

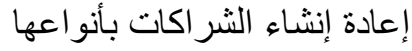

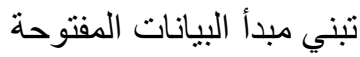

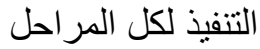

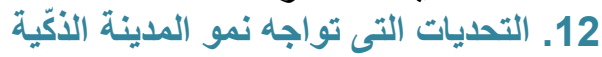

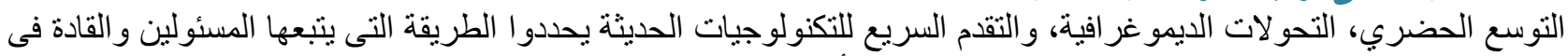

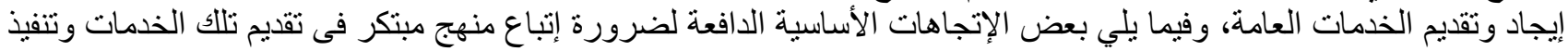

المهام المطلوبة في المدينة (Ruthbea 2013) :

$$
\text { زيادة المنافسة العالمية بين المدن لتقديم الموادئ الهب (الإبتكار ات). }
$$

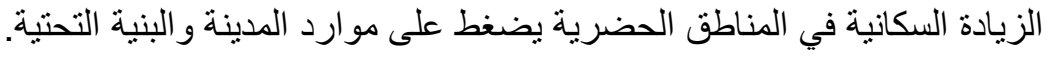

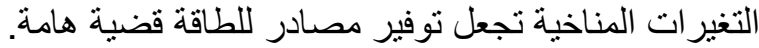

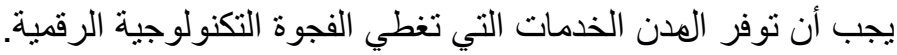

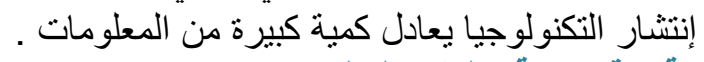

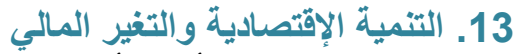

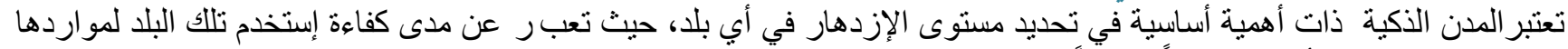

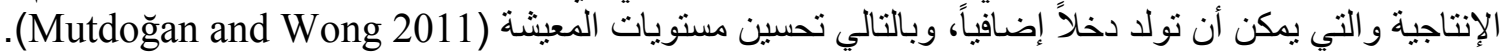

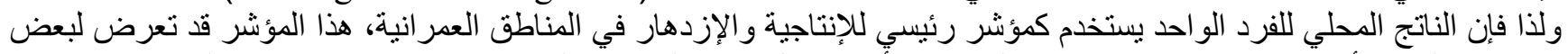

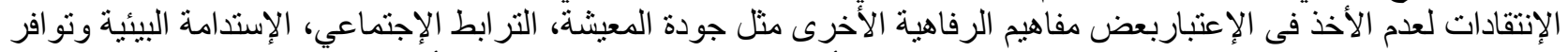

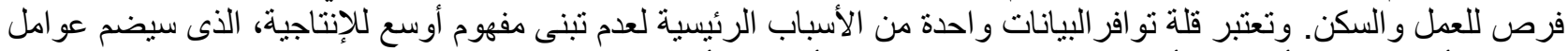

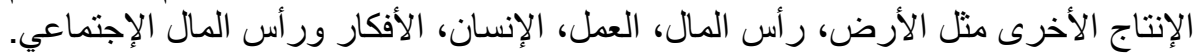

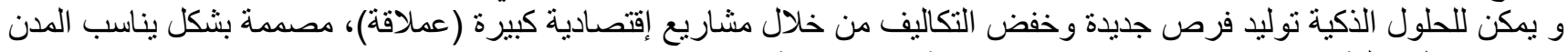

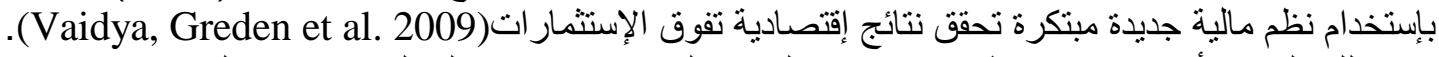

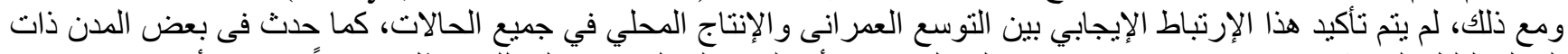

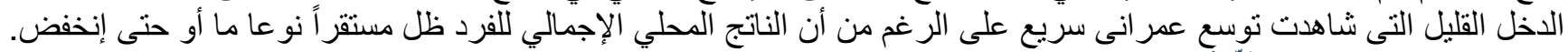

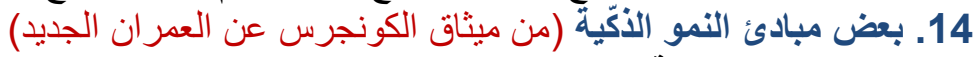

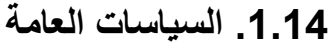

التخطيط بالتعاون بين البلديات المختلفة داخل كل منطقة.

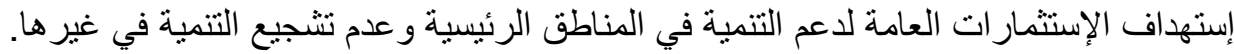

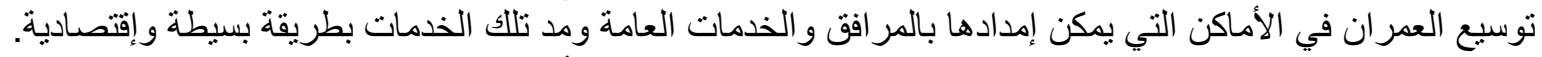

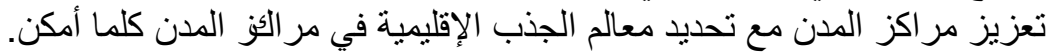
عمل تطور ات تتمانشي مع التنوع الحالي مع إستخدام الموارد المتجددة في الإقتصاد المحلي. 
تفعيل قرارات التطوير مع إنشر الك الجهات المعنية (المجتمع و المو اطنين) في عملية صنع القرار. تقديم الحو اقز وإز الة الحواجز التشريعية لتمكين المطورين من إتخاذ القرارات الصني الصحيحة.

2.14. إستراتيجيات التخطيط

ضرورة تنوع إستخدام الأر اضي و إعادة تخطيط خطوط النقل لتقليل المسافات التى تقطعها السيار ات مع نوفير مجموعة من خيار ات النقل لتخفيف الإزدحام (Strategic Planning Department 2009).

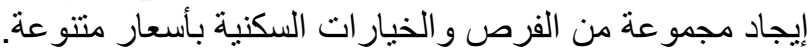

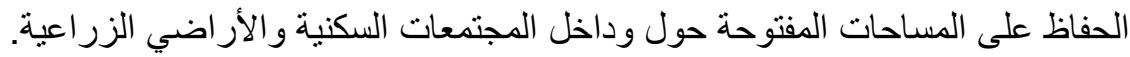

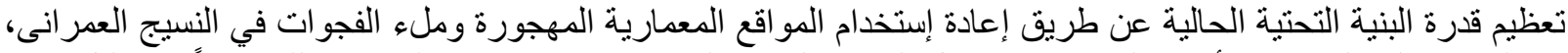
مع الحفاظ على المباني والأحياء التاريخية وتحويل المئية المباني القديمة إلى إستخدامات جديدة كلما كان ذلك ممكناً مع تقليل

تعزيز الثعور بالإنتماء للمكان بإعتباره لبنة بناء للتنمية المجتمعية. 3.14 مفاهيم التصميم العمراني

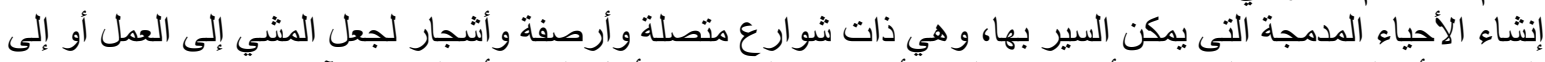

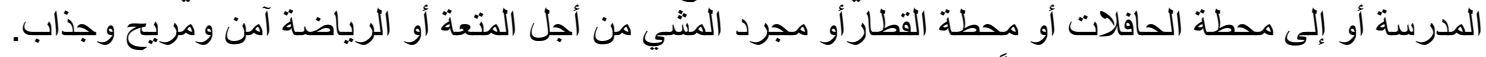

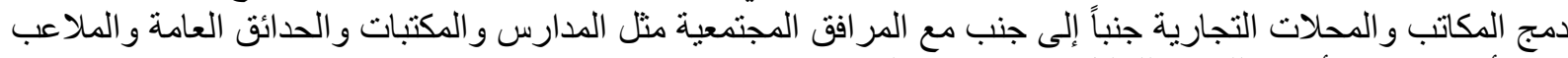
في الأحياء لإنثاء أماكن للمشي للتقليل من إستخدام السيار التهات.

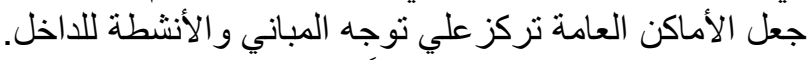

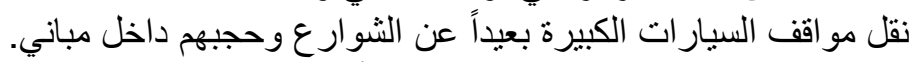

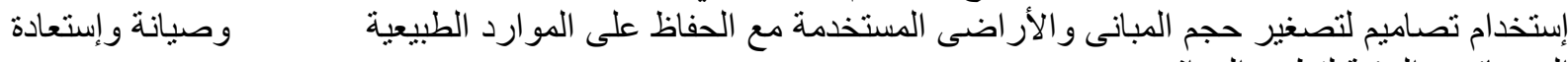

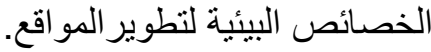

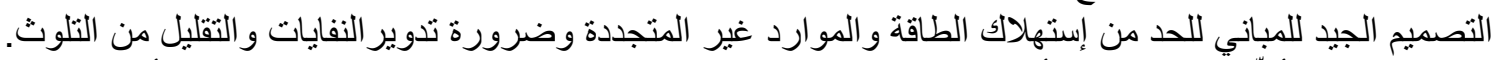

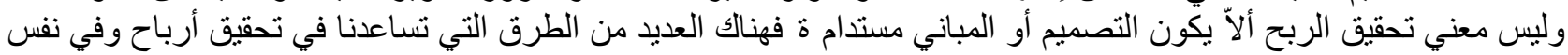
الوقت نستطيع الحفاظ علي إستدامة المباني منها:

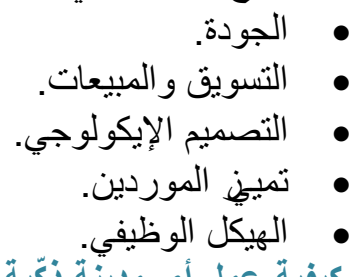

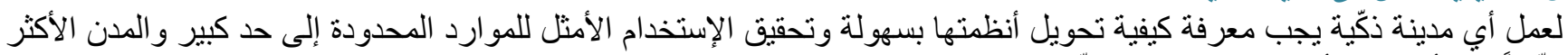

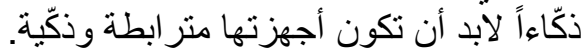

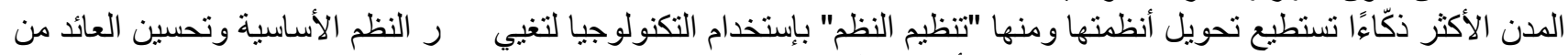

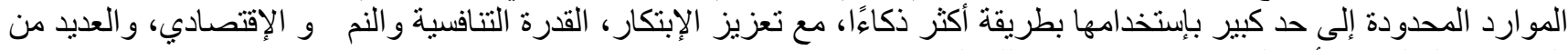

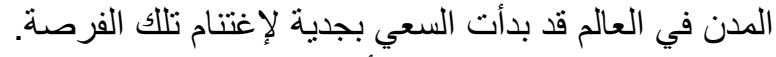

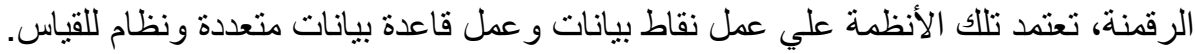

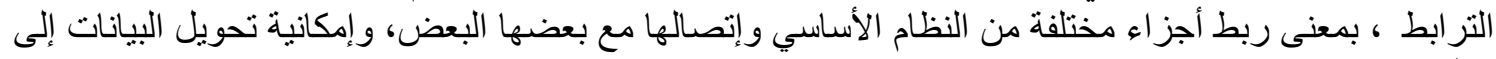
معلومات.

القدرة على إستخدام المعلومات التي تم إنشاؤها، وترجمتها إلى المعرفة الحقيقية، مما يسمح بإجر اءات واعية ومفيدة.

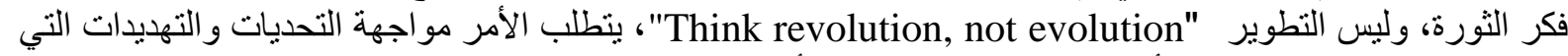

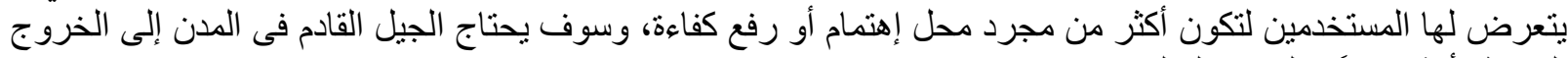
إلى نظم أكثر ذكاءًا بالتغيري الفعلي.

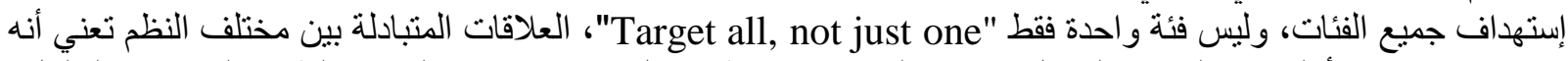
لابد من وضوح الأولويات وليس هناك حل واحد مثالي لابد من تطبيق بل لابد من إناحة الفرصة لطرح العديد من الحلّول 


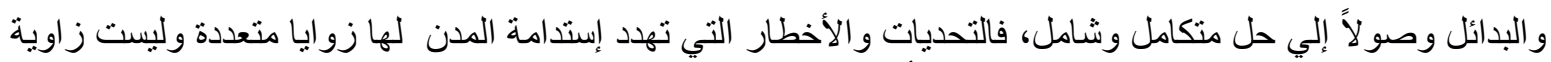

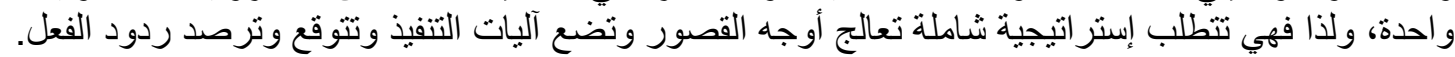

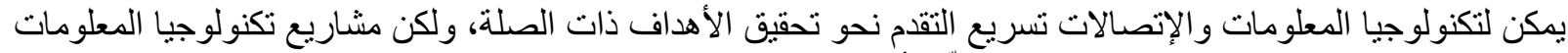

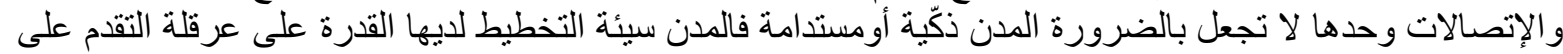

المدى البعيد (Lankhorst 2009).

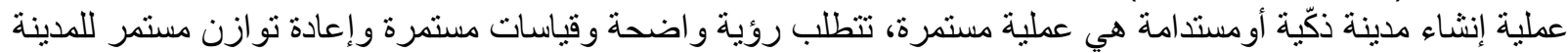

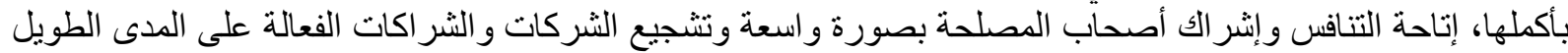

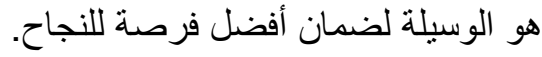

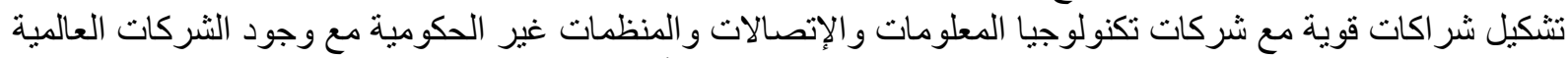

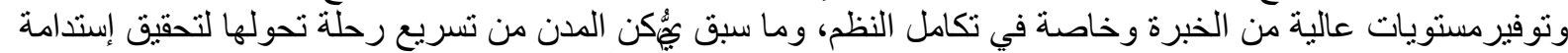

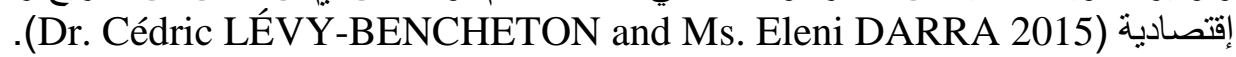
تم إقتر اح تصميم تللك المنطقة لترتبط بوسط المدينة ولتكون نموذج مصغر لمدينة تجمع بين تكنولوجيا المعلومات و الإتصالات و الإبتكار. نظم الإستجابة لحالات الإن الطوارئ الإن على أساس مركز العمليات المتكاملة، شبكة الألياف الضوئية، البنية التحتية، و المر اقبات المرئية والخدمات الإنتجابة المدارة.

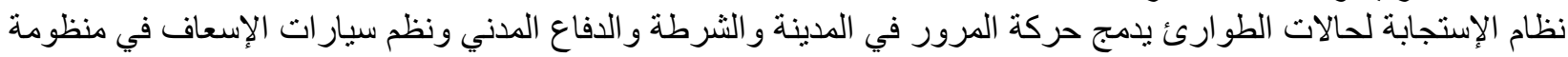

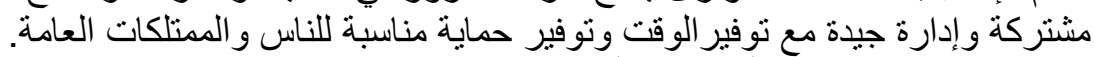

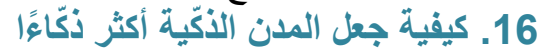

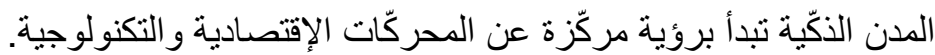
الرؤية الناجحة للمدينة يجب النابة تعالج أربعة محركات إقتصادية وهي (Adie and Robert 2014): ل الأولى هى الإنتاجية.

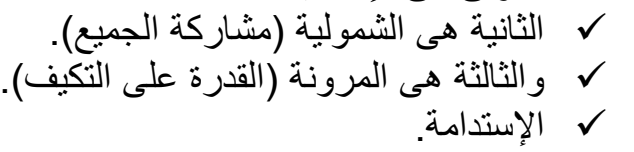
يجب أن تعبد المدن بناء (إصلاح) النظم الحكومية لتقوم بتنفيذ الرؤى الإقتصادية بنجاح.

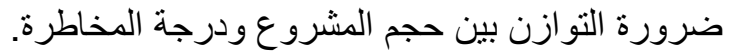

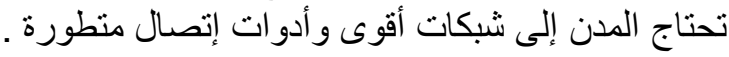

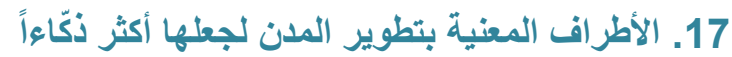

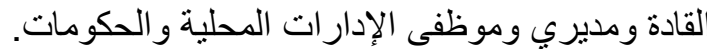

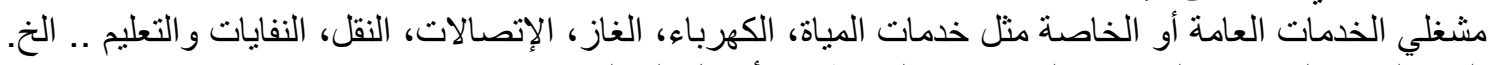

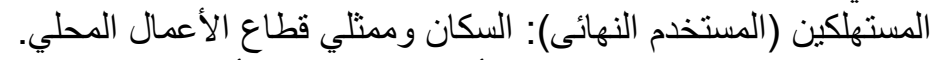

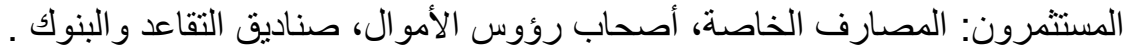

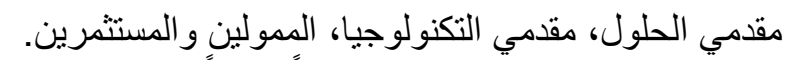

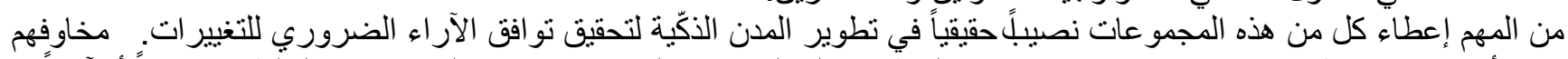

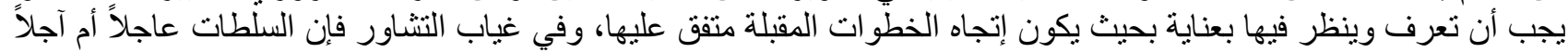

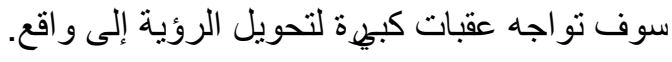

18. رؤى تشهل تقعيل المدن الأكّية هنالك العديد من الرؤى التى وضعت من العديد من العلماء و المهتمين بتفعيل تلك الرؤى إرتكزت على ما يلى :

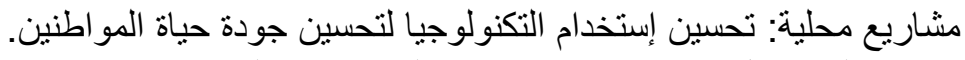

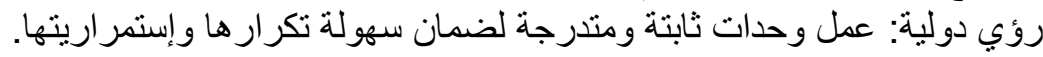
تكنولوجيا قياسية: تطوير برونوكول المدينة لتحديد كيف ينبغي وضع معايير التكنولوجيا بنديا بدقة للمدن. 
أصبح المو اطنين يقدمون الخدمات وليس مستخدمين لها فقط، لللك يجب وضع خطي خطة جيدة للمشتركين، المدخلات و الأفكار من الأطر اف المعنية و على نطاق واسع داخل المدينة و هذا يعني أن تخطيط المدن يحتاج لعمليات تحديث جذئ جذرية.

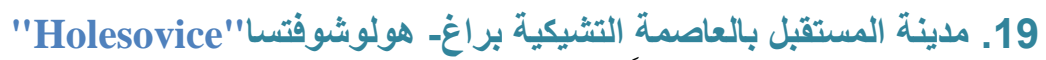

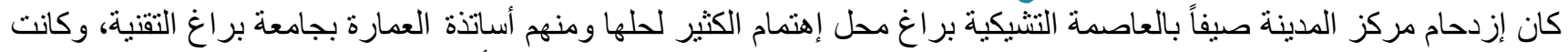

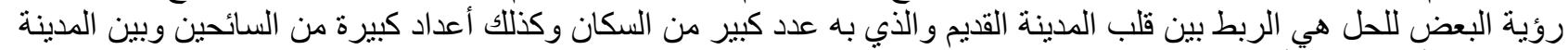

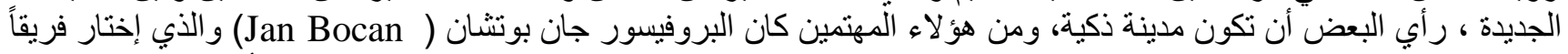

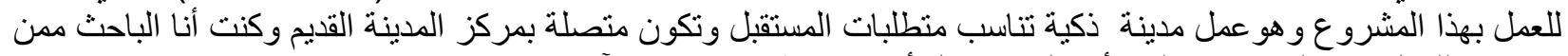

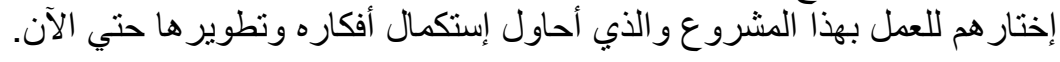

وقد أعدت كل مجمو عة من الفريق حلو لاً وتصميمات عديدة ممزوجة بأفكار حديثة ورؤي تناسب متطلبات المستقبل وقابلة للتنفيذ،

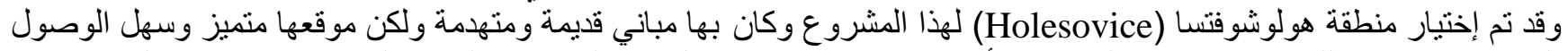

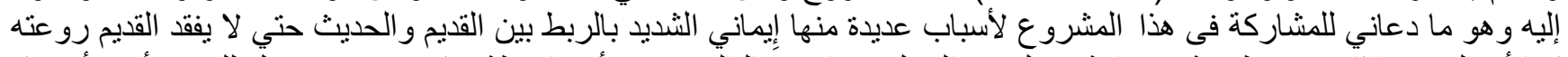

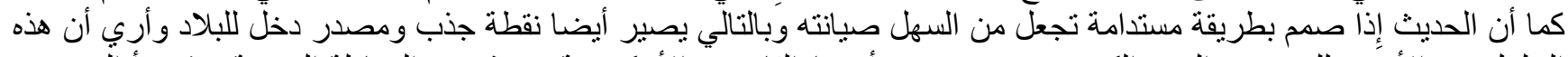

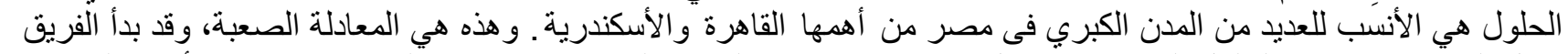

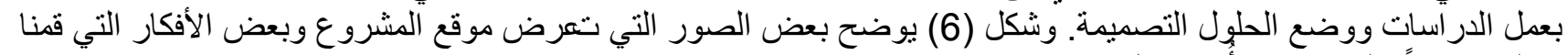

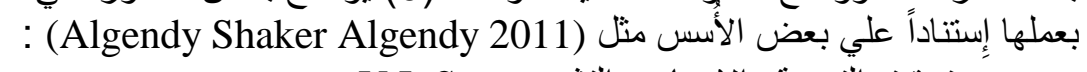

V.L.S تحقيق التهوية والإضـاءة والتشميس الإنيس

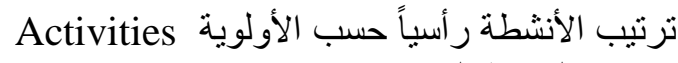

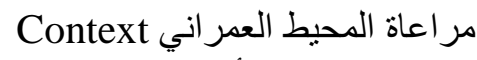

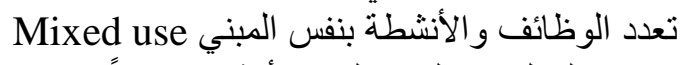

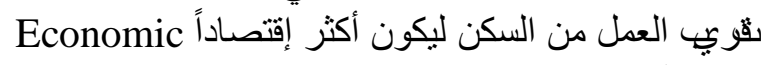

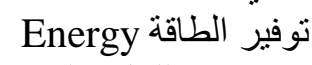

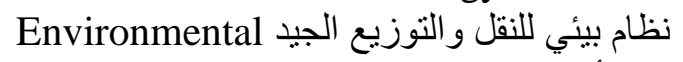

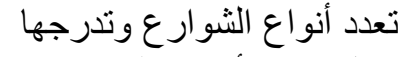

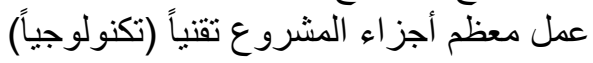

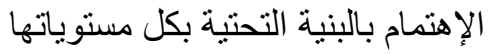

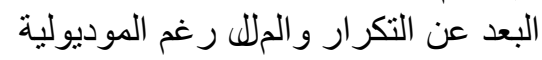

$$
\begin{aligned}
& \text { الربط الوظيفي بين المديثة القديمة والمبر المقترحة }
\end{aligned}
$$

ثم قمنا بالتعديل مر ات ومر ات حتي تم الإتفاق علي العديد من الأسس وتجميع العديد من المزايا ونقاط الجذب لمحاولة وضع أكبر

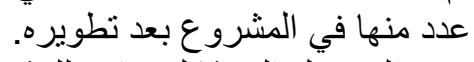

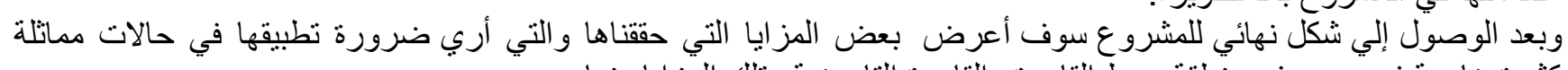

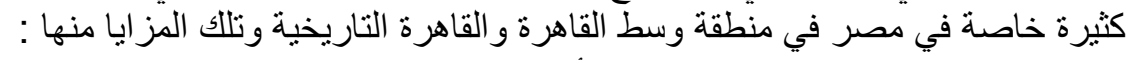

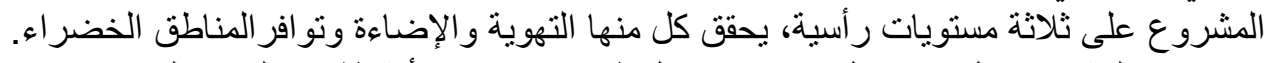

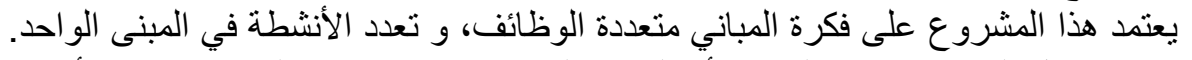

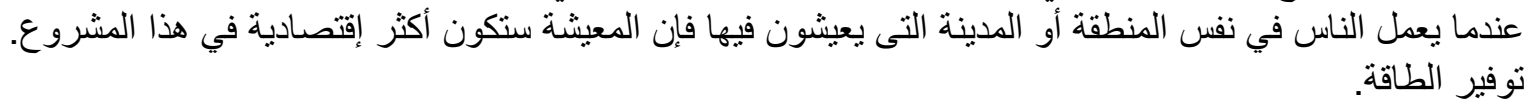



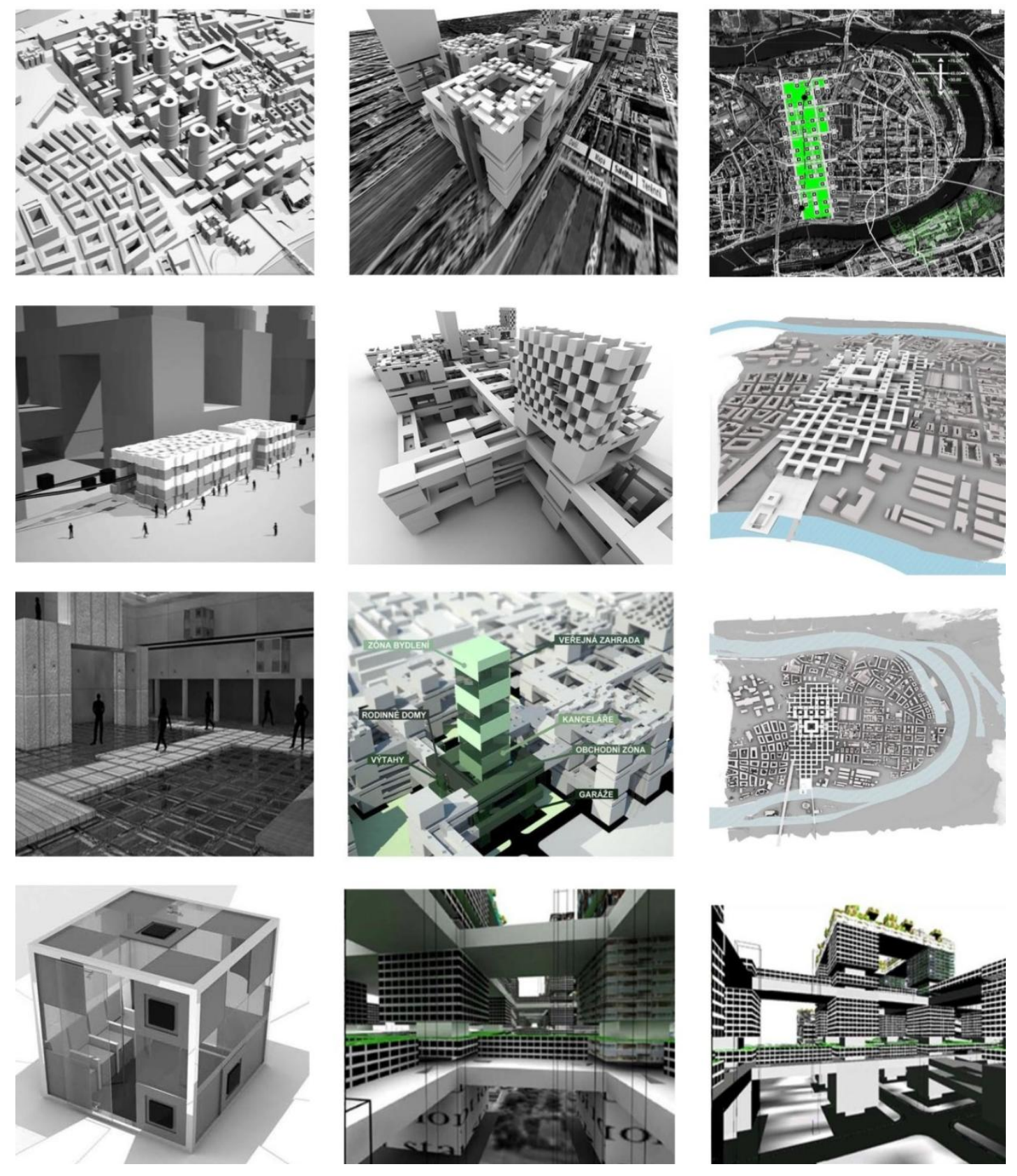

شكل ( 7 ) مجموعة صور توضح موقع مشروع هولوشوفتسا ”Holesovice“ وبعض الأفكار المقترحة من فريق العمل لمدينة مستقبل ذكّية المصدر: بتصرف من الباحث

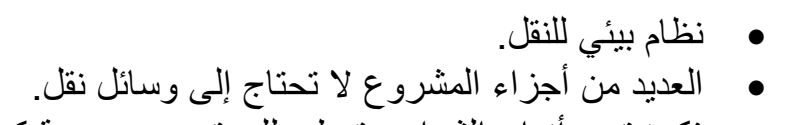

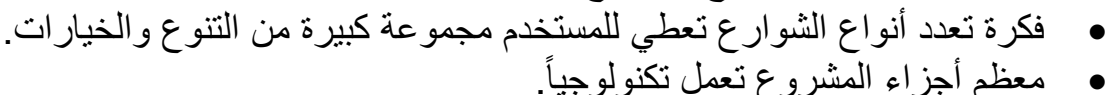

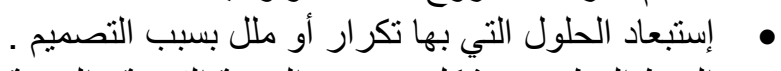

• الربط الوظيفي وبشكل جيد بين المدينة القديمة و المدينة المقترحة .

أمّا بعض العيوب التي تم التظلب عليها فمنها : 
• • •

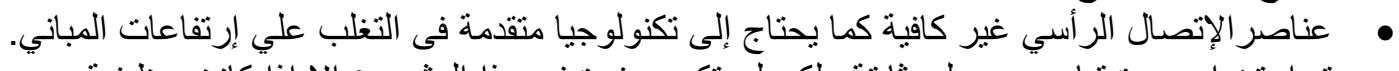

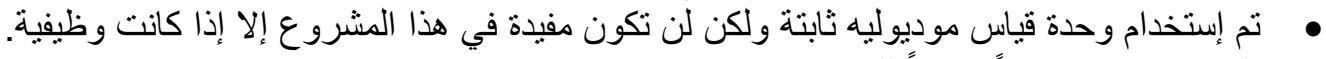

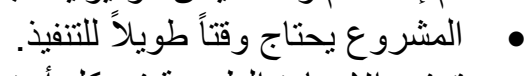

• • • • • •

بعد در اسة المدن الذكّية ومتطلباتها وتحدياتها وكيفية بناءها توصل الباحث بعد الإستعانة ببعض النتائج التي توصل إليها عدد من الباحثيين منهم مؤسسة International Electro technical Commission IEC بنتائجها لوضع تللك المعايير إلي بعض الحلول الذكية وذات القيمة والتي يمكن إستخدامها كمصفوفة للتقبيم و التي تقسم إلي خمس

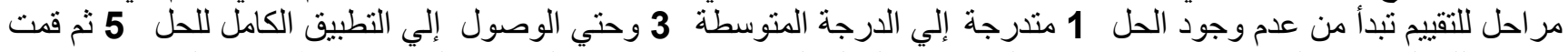

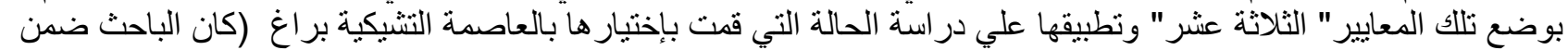

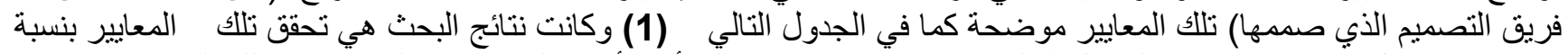

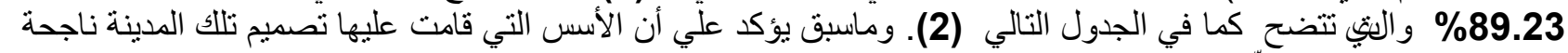


نحو مدن ذكية ذات كفاءة وظيفية در اسة تطبيقية علي مشروع مدينة المستقبل بالعاصمة التشيكية بر اغ

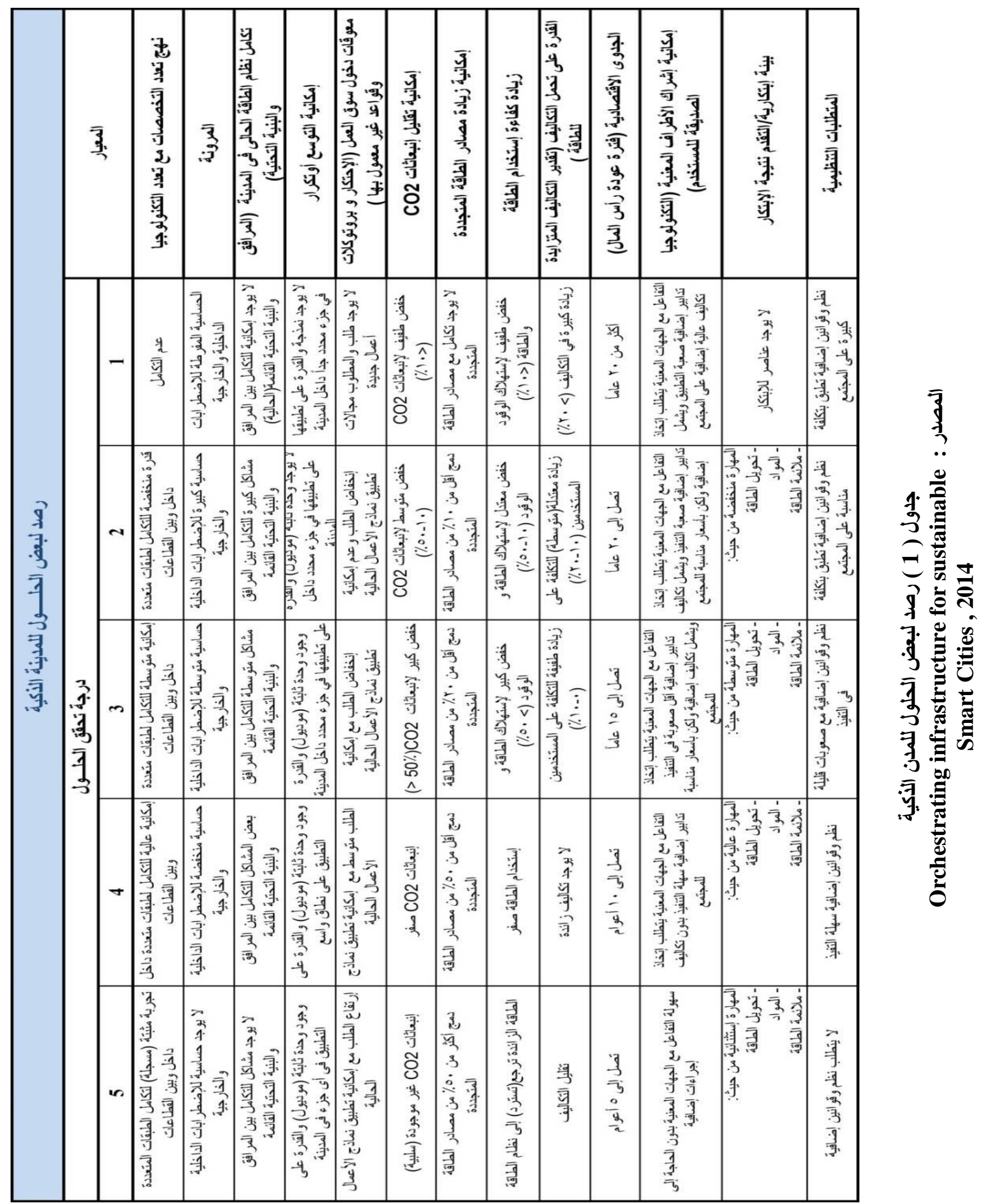


نحو مدن ذكية ذات كفاءة وظيفية دراسة تطبيقية علي مشروع مدينة المستقبل بالعاصمة التشيكية بر اغ

\begin{tabular}{|c|c|c|c|c|c|c|c|}
\hline \multirow{2}{*}{ a } & \multirow{2}{*}{ المعيــــار } & \multicolumn{5}{|c|}{ مدى تحقيق المشروع للأهداف } & \\
\hline & & 1 & 2 & 3 & 4 & 5 & \\
\hline 1 & نهج تعدد التخصصات مع تعدد التكنولوجيا & & & & & $\mathrm{v}$ & $100 \%$ \\
\hline 2 & 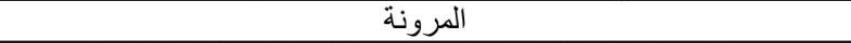 & & & & & $\mathrm{v}$ & $100 \%$ \\
\hline 3 & تكامل نظام الطاقة الحالى فى المدينة (المر افق و البنية التحتية) & & & & $\sqrt{ }$ & 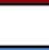 & $80 \%$ \\
\hline 4 & إمكانية التوسع أوتكرار & & & & & $\sqrt{ }$ & $100 \%$ \\
\hline 5 & معوقات دخول سوق العمل (الإحتكار وبروتوكلات وقو اعد غير معمول بها ) & & & & & $\sqrt{ }$ & $100 \%$ \\
\hline 6 & إمكانية تقليل إنبعاثات CO2 & & & $\mathrm{v}$ & & & $60 \%$ \\
\hline 7 & إمكانية زيادة مصادر الطاقة المتجددة & & & & & $\bar{v}$ & $100 \%$ \\
\hline 8 & زيادة كفاءة إستخدام الطاقة & & & & $\sqrt{ }$ & & $80 \%$ \\
\hline 9 & القدرة على تحمل التكاليف (تقدير التكاليف المتز ايدة للطاقة ) & & & & $\checkmark$ & & $80 \%$ \\
\hline 10 & الجدوى الإقتصادية (فترة عودة رأس المال) & & & & & $\bar{v}$ & $100 \%$ \\
\hline 11 & إمكانية إشر الك الأطر اف المعنية مستخدمأ التكنولوجيا الصديقة للمستخدم & & & & & $\sqrt{ }$ & $100 \%$ \\
\hline 12 & بيئة إبتكارية/التقدم نتيجة الإبتكار & & & & $\sqrt{ }$ & & $80 \%$ \\
\hline 13 & المتطلبات التنظيمية & & & & $\sqrt{ }$ & & $80 \%$ \\
\hline
\end{tabular}

1. Adie, T. and P. Robert (2014). GETTING SMARTER ABOUT SMART CITIES. Washington, ESADE.

2. Alan, C., Q. Nguyen and S. Henry (2006). Urban Development Strategy.

3. Algendy Shaker Algendy (2011). PHD,An Approach Towards Evaluation of The Functional Efficiency of Office Buildings Prague, Department of economics and managment in civil enginnering

4. Dr. Cédric LÉVY-BENCHETON and Ms. Eleni DARRA (2015). Cyber security for Smart Cities. European Union, European Union Agency For Network And Information Security.

5. Federal Ministry of Economics and Technology (2010). ICT Strategy of the German Federal Government: Digital Germany 2015, Federal Ministry of Economics and،Technology،(Public Relations, Berlin.

6. Giffinger, R., C. Fertner, H. Kramar 'R. Kalasek, N. Pichler and E. Meijers (2007). Smart cities, Ranking of European medium-sized cities. Vienna, Asset One Immobilienentwicklungs AG, Kaiserfeldgasse 2, 8010 Graz.

7. International Electrotechnical Commission IEC (2014). Orchestrating infrastructure for sustainable Smart Cities. Switzerland, International Electrotechnical Commission IEC.

8. Lankhorst, M. (2009). Introduction to Enterprise Architecture. Enterprise Architecture at Work, Springer Berlin Heidelberg: 1-11.

9. Liam, M. (2010). Smart Cities and Sustainable Technology. U.S.A, Siemens Ltd.

10. Macmillan, S. (2004). Designing better buildings : quality and value in the built environment. New York, Spon.

11. Mutdoğan, S. and T.-C. Wong (2011). Towards Sustainable Architecture: The Transformation of the Built Environment in İstanbul, Turkey. Eco-city Planning. T.-C. Wong and B. Yuen, Springer Netherlands: 239260.

12. Ruthbea, Y. C. (2013). Smart Cities and the Internet of Everything: The Foundation for Delivering NextGeneration Citizen Services, Cisco.

13. School, o. A. P. and I. o. T. Massachusetts (2008). Sustainable Housing and Community Design Jaban.

14. Shroff, G. (2010). Enterprise cloud computing : technology, architecture, applications. Cambridge ; New York, Cambridge University Press.

15. Strategic Planning Department (2009). PROGRAMME FOR THE IMPLEMENTATION OF THE PRAGUE STRATEGIC PLAN. The City of Prague.

16. Vaidya, P., L. Greden 'D. Eijadi, T. McDougall and R. Cole (2009). "Integrated cost-estimation methodology to support high-performance building design." Energy Efficiency 2(1): 69-85.

17. Zahariadis, T. a. P., Dimitri (2011) "Towards a Future Internet Architecture." The enterprise engineering series.

World Wide Web sites

http://viralvibes.net/everything-you-wanted-to-know-about-smart-cities

http://tzm-arabic.com/archives www.bmwi.de. 